\title{
EVALUATION OF INTERNAL ADAPTATION OF FULL CONTOUR ZIRCONIA CROWNS VERSUS VENEERED ZIRCONIA CROWNS - IN VITRO STUDY
}

\begin{abstract}
Abdulsattar Hasan Al-baadani*, Rana Mahmoud Sherief** and Ashraf Taher Kheireldean***
\end{abstract}
\begin{abstract}
Purpose: To evaluate the internal adaptation of full contour zirconia crowns to veneered zirconia with two different finish line designs (shoulder and deep chamfer).

Materials and Methods: Twenty four dies were received twelve full contour zirconia crowns (Prettau) and twelve veneered zirconia crowns (ICE Zirconia). Each group will be subdivided into two equally subgroups: with Shoulder finish line and with Deep chamfer finish line. Measurements of internal fitness were recorded.
\end{abstract}

Results: The lowest overall mean value of internal adaptation was recorded with Ice zirconia crowns with deep chamfer finish line design $(74.6 \mu \mathrm{m} \pm 19.14)$, then with shoulder finish line design $(93.40 \mu \mathrm{m} \pm 9.99)$, followed by Prettau zirconia crowns with deep chamfer finish line design $(157 \mu \mathrm{m} \pm 27.25)$ and finally Prettau zirconia crowns with shoulder finish line design $(181 \mu \mathrm{m} \pm$ 24.86).

Conclusions: The ice zirconia crowns recorded better overall internal adaptation values than Prettau crowns with both finish line designs.

KEY WORDS: Internal Adaptation, fit, full contour.

\section{INTRODUCTION}

Nowadays prevalence of interfacial failures of zirconia crowns led to the clinical application of full contour zirconia. However, some properties of monolithic zirconia need to be improved such as relative opacity, monochromatic appearance, and marginal adaptation after polishing and glazing. ${ }^{1}$ The fit of zirconia veneered copings or full contour zirconia varies with the variation in the finish line design and preparation angles. With monolithic zirconia, a preparation can be done with reduction very similar to that of fullgold restorations. An occlusal clearance of 1.0 to $1.5 \mathrm{~mm}$ is recommended. Feather-edge margins are acceptable depending upon laboratory team's skill. ${ }^{2}$ There are a lot of researches and studies that has been done with different types of finish lines.

* B.D.S., Master Degree in Fixed Prosthodontics, Faculty of Oral and Dental Medicine, Cairo University, Egypt.

** Assistant Professor of Fixed Prosthodontics, Faculty of Oral and Dental Medicine, Cairo University. 
Some studies fabricated zirconia FPD restorations with shoulder finish line ${ }^{3}$, Other studied fabricated it with chamfer finish line ${ }^{4}$ Or both of them (shoulder and chamfer finish lines). ${ }^{5}$ Therefore this study will assess internal fit adaptation of full contour zirconia restoration relative to zirconia coping with porcelain veneer with two different finish line designs.

\section{MATERALS AND METHODS}

Two specially designed stainless steel dies were machined in a standardized manner using an engineering lather machine. A $90^{\circ}$ shoulder finish line of $1 \mathrm{~mm}$ thickness was prepared in one die and chamfer finish of $1 \mathrm{~mm}$ thickness was prepared in other. 24 Impressions were made; giving a total number of $\mathbf{1 2}$ impressions for each master dies. The impressions were then poured into type IV improved dental stone. The Roland Milling Unit was used to mill the Prettau blocks to produce the CAD/CAM milled full contour crowns. Each stone die was scanned with Zirkonzhan scanner. The full anatomical crown was designed, $(1.5 \mathrm{~mm})$ in thickness and each crown was given a number in the software that corresponds to its scanned stone working die. The fabrication of Ice zirconia copings were similar as mentioned previously to the construction of full contour zirconia except the dimension of the Ice blocks was (98 x $10 \mathrm{~mm}$ ) rather than $(98 \times 14)$ in Prettau blocks. Veneering of the cores were done using the layering technique by one specialist technician.

\section{Measurement of Internal Adaptation}

A light-body silicon rubber impression paste was used for the purpose of recording the cement space and the metal die. A light-body material was injected in the fitting surface of the crown and positioned in the corresponding metal die with the constant defined load of $40 \mathrm{~N}$ for 3 minutes using the loading device. The crowns were boxed with pink wax and to support the thin light silicon impression film, heavy body silicon was injected onto the light body at the fitting surface of the crown so as to adhere to the light body film and form one piece with it creating the silicon die replica. After setting of the supporting heavy-body material, each silicon die replica was removed from the crown and carefully segmented with a surgical blade no. 11 in mesiodistal direction. The following are the five points that were defined for internal adaptation : P1, left axial cervical thirds; P2, left axial occlusal third; P3, middle occlusal; P4, right axial occlusal third and P5 right axial cervical third. Measurements were recorded by viewing the specimens at $12.5 \mathrm{X}$ magnification under stereomicroscope.

\section{RESULTS}

\section{Revealed}

It was found that the lowest overall mean of internal adaptation values was recorded for prettau zirconia crowns with a deep chamfer finish line (157 $\pm 27.25 \mu \mathrm{m})$. Prettau zirconia crowns with shoulder finish line design recorded higher values $(181 \pm$ $24.86 \mu \mathrm{m})$. But the differences was statistically insignificant $(p>0.05)$ between the two finish line designs. For the ice zirconia crowns it was found that the lowest overall mean of internal adaptation values was recorded with the deep chamfer finish line $(74.6 \pm 19.14 \mu \mathrm{m})$, while with shoulder finish line design recorded higher values $(93.40 \pm 9.99 \mu \mathrm{m})$. But the difference was statistically insignificant ( $p>0.005$ ) between the two finish line designs.

Prettau crowns with deep chamfer finish line design $(157 \pm 27.25 \mu \mathrm{m})$ showed a higher internal adaptation value when compared to the ice zirconia crowns with deep chamfer finish line design $(74.6 \pm 19.14 \mu \mathrm{m})$. A statistically significant difference ( $\mathrm{p} \leq 0.001$ ) was observed between the two restorative systems with the deep chamfer finish line design Table (2). Whereas with the shoulder finish line design, prettau crowns $(181 \pm 24.86 \mu \mathrm{m})$ showed a higher internal adaptation value when compared to ice zirconia crowns $(93.40 \pm 9.99$ $\mu \mathrm{m})$ with shoulder finish line, indicating better internal adaptation for the ice zirconia crowns. 
There was statistically significant difference $(\mathrm{p} \leq 0.001)$ between the two restorative systems with the shoulder finish line design.

\section{DISCUSSION}

Additional silicon impression was used to fabricate impression die replicas of the master dies for internal adaptation measurements according to Tsitrou et $\mathbf{a l}^{6}$ different techniques had been proposed for internal adaptation measurements which include optical microscope, micro computed tomography, stereomicroscope and replica technique with a magnification microscope. Regarding the effect of the finish line design on the internal adaptation of both restorative systems, results in this study reviled that the overall mean value of the internal adaptation recorded for prettau zirconia crowns with deep chamfer finish line design were lower than prettau zirconia crowns with shoulder finish line design. When comparing the internal adaptation of the two crown materials within each different finish line designs, prettau zirconia crowns with both deep chamfer and shoulder finish line designs recorded higher overall mean internal adaptation values when compared to ice zirconia crowns with both deep chamfer and shoulder finish line designs. Collectively our results indicated that crowns with deep chamfer finish line design showed lower overall mean value of internal adaptation than crowns with shoulder finish line design.

\section{CONCLUSIONS}

Within the limitations of this study, the following conclusions can be drawn:

1. The overall mean values of the internal adaptation of the two restorative systems were within the clinical acceptance range.

2. The ice zirconia crowns recorded better overall internal adaptation values when compared to prettau crowns with both finish line designs.

\section{REFERENCES}

1 Kim K-Y, Kwon T-K, Kang T-J, Yang J-H, Lee S-J, Yeo I-S. Digital veneering system enhances microtensile bond strength at zirconia coreveneer interface. Dent Mater J 2014; 33: 792-798.

2 Jack D. G. Tooth in a Bag : Same-Day Monolithic Zirconia Crown. Dent Today 2013; 2: 2-7.

3 Takashi Miyazaki, Takashi Nakamura, Hideo Matsumura, Seiji TK. ScienceDirect Current status of zirconia restoration. J Prosthodont Res 2013; 57: 236-261.

$4 \mathrm{Ng} \mathrm{J}$, Ruse D, Wyatt C. A comparison of the marginal fit of crowns fabricated with digital and conventional methods. J Prosthet Dent 2014; 112: 555-60.

5 Baig MR, Tan KB, Nicholls JI. Evaluation of the marginal fit of a zirconia ceramic computer-aided machined (CAM) crown system. J Prosthet Dent 2010; 104: 216-227.

6 Effrosyni A. Tsitrou, Northeast S. VNR. Evaluation of the internal fit of three marginal designs of resin composite crownw using CAD/CAM. J Prosthet Dent 2007; 35:68-73. 